\title{
ANÁLISE DE ELEMENTOS FINITOS PARA PROBLEMAS DE VIBRAÇÕES EM PASSARELAS COMPOSTAS POR VIGAS MISTAS COM FUROS NA ALMA DEVI- DO AO CAMINHAR DE PEDESTRES
}

\author{
F. F. Feitosa ${ }^{1}$, J. G. S. da Silva ${ }^{2}$, L. R. O de Lima², P. C. G. da S. Vellasco ${ }^{2}$ \\ ${ }^{1}$ Programa de Pós-Graduação em Engenharia Civil, PGECIV, Universidade do Estado do Rio \\ de Janeiro, UERJ (eng.bm@hotmail.com)
}

${ }^{2}$ Departamento de Estruturas e Fundações, Universidade do Estado do Rio de Janeiro, UERJ

\begin{abstract}
Resumo. Limitações de altura têm sido impostas sobre edificações por regulamentos de zoneamento urbano e aspectos econômicos e estéticos. Para se proporcionar a passagem de tubulações de grande diâmetro sob vigas metálicas, um pé-direito alto é normalmente requerido. Uma solução frequentemente utilizada em projeto diz respeito à abertura de furos na alma das vigas de aço para passagem das tubulações de serviço. Considerando-se os aspectos abordados anteriormente, este trabalho de pesquisa tem por objetivo a avaliação da resposta dinâmica de passarelas de pedestres, onde o projeto estrutural prevê a utilização de vigas celulares caracterizadas por aberturas circulares na alma. Pretende-se, portanto, verificar a influência das aberturas nas almas dessas vigas sobre a resposta dinâmica das passarelas. As ações dinâmicas representativas do caminhar dos pedestres são simuladas por meio de um modelo matemático que considera uma descrição espacial e temporal e, ainda, inclui o efeito do impacto do calcanhar humano. Os modelos estruturais investigados correspondem a passarelas mistas (aço-concreto) com 10m a 30m de extensão. São empregadas técnicas usuais de discretização, via método dos elementos finitos, por meio do programa ANSYS. A resposta dinâmica dos modelos, em termos dos deslocamentos e acelerações, é obtida na fase permanente da resposta para duas situações distintas: vigas de alma cheia e vigas celulares obtidas a partir destas de alma cheia. Uma avaliação crítica, qualitativa e quantitativa, sobre a resposta dinâmica das passarelas possibilita verificar a influência dos furos nas almas das vigas metálicas, no que diz respeito ao conforto humano, mediante comparações com códigos e recomendações de projeto.
\end{abstract}

Palavras-chave: Passarelas de pedestres, Dinâmica estrutural, Conforto humano.

\section{INTRODUÇÃO}

Atualmente, são frequentes as limitações de “pé-direito” em edificações de vários pavimentos devido às leis de zoneamento e as exigências econômicas, além das considerações sobre a estética da estrutura. Normalmente, grandes espaços são necessários para permitir a passagem de tubos e dutos sob as vigas de aço, o que consequentemente, pode prejudicar o aproveitamento do espaço entre o piso e o teto. 
Inúmeras soluções podem ser utilizadas para superar esses problemas, tais como vigas misuladas e treliças. Contudo, a solução mais adotada nos países de primeiro mundo é a utilização de aberturas na alma das vigas de aço. Nesses casos, os perfis das vigas de aço podem apresentar aberturas circulares ao longo de todo o seu comprimento, e estas são denominadas vigas celulares. Outras formas de abertura na alma das vigas podem ser utilizadas, tal como a hexagonal e, neste caso, estas são denominadas por vigas casteladas [1].

Geralmente, as passarelas de pedestres mistas (aço-concreto) são constituídas por longarinas e transversinas de aço e o piso é composto por lajes de concreto armado. Por outro lado, este tipo de sistema estrutural vem se tornando um marco arquitetônico relevante em grandes áreas urbanas. Nos últimos anos, devido ao avanço tecnológico, as passarelas têm sido projetadas com características bastante esbeltas, o que tem conduzido a estruturas com baixos valores de frequência e amortecimento estrutural [1-6].

Assim sendo, as frequências das ações dinâmicas associadas aos passos dos pedestres (caminhando ou correndo) podem coincidir com a frequência fundamental da estrutura, gerando o fenômeno físico da ressonância, e, desta forma, os efeitos dinâmicos não podem ser negligenciados. Deste modo, este trabalho de pesquisa foi desenvolvido de modo a avaliar o comportamento dinâmico de passarelas de pedestres mistas (aço-concreto), nas quais as vigas de aço possuem aberturas na alma de diferentes geometrias e formas. Objetiva-se verificar a influência das aberturas nas almas dessas vigas sobre a resposta dinâmica das passarelas.

Os modelos estruturais investigados correspondem a passarelas mistas de $10 \mathrm{~m}$ a $30 \mathrm{~m}$ de extensão. O sistema estrutural utilizado para a travessia de pedestres é composto por perfis metálicos de seção "I” e um tabuleiro de concreto armado. A análise fundamenta-se na modelagem numérico-computacional dessas passarelas. Para tal, são empregadas técnicas usuais de discretização, via utilização do programa ANSYS [7]. Uma avaliação crítica da resposta dinâmica das passarelas possibilita verificar a influência das aberturas nas almas das vigas, mediante comparações com limites de normas de projeto.

Para efeito deste estudo, além do perfil de alma cheia, a análise foi efetuada sobre duas vigas celulares fabricadas a partir de um mesmo perfil VS soldado, no qual são efetuados dois cortes longitudinais formando módulos contínuos de semicircunferência intercalados por um pequeno segmento reto (Fig. 1a). Posteriormente, as duas metades são separadas e sobrepostas de forma a serem soldadas as partes retas (Fig. 1b). A título de exemplo, considerando-se uma passarela com 25m de vão, duas longarinas da estrutura, associadas a vigas de aço celulares foram modeladas de acordo com o número mínimo e máximo de aberturas possíveis (16 e 31 aberturas, respectivamente), no que diz respeito ao perfil gerado após o corte, e de acordo com as características geométricas do perfil de alma cheia inicial.

É importante ressaltar que as vigas celulares podem ser produzidas com inúmeras combinações entre a altura da seção transversal, diâmetro da abertura e distância entre os centros de aberturas. Na sequência do estudo, a Fig. 2 apresenta uma combinação consagrada internacionalmente e que tem sido bastante utilizada na prática corrente de projeto, pelo fato de oferecer resultados compensadores [8]. 


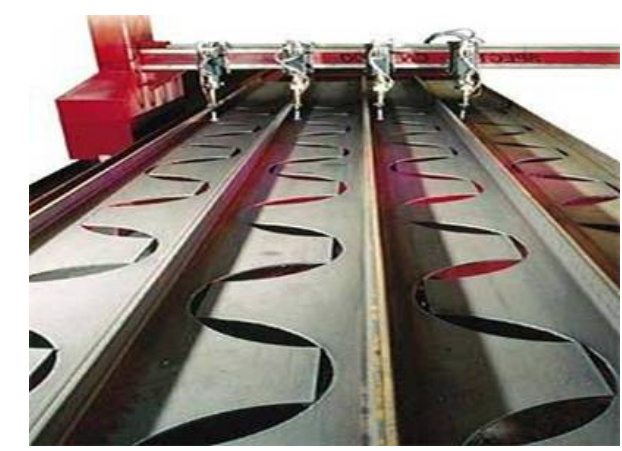

(a) Corte da alma

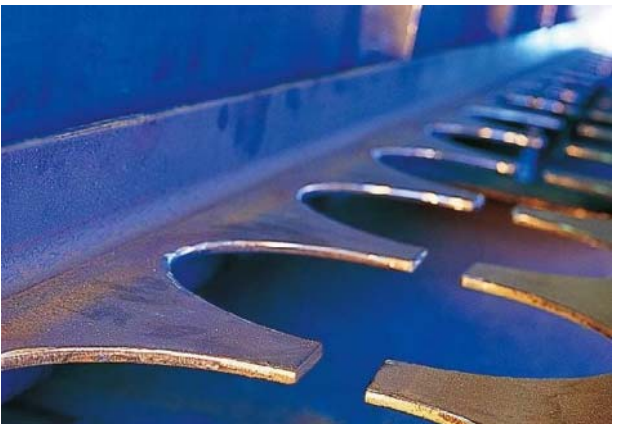

(b) Preparação para soldagem

Figura 1. Fabricação de vigas celulares (http://www.arcellormittal.com).
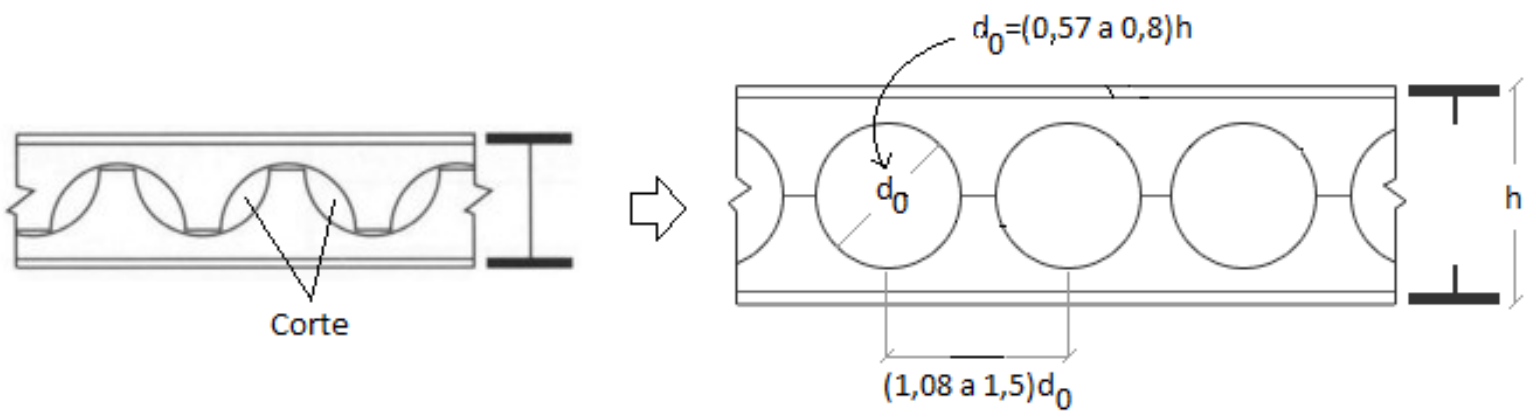

Figura 2. Geometria do diâmetro e do espaçamento para a fabricação das vigas celulares [8].

\section{MODELAGEM DO CAMINHAR HUMANO}

A presente análise baseia-se em um modelo mais realista para representar a excitação dinâmica proveniente do pedestre, de forma a incorporar o impacto transiente do calcanhar humano devido ao caminhar. Neste modelo de carregamento, o movimento de pernas que causa a subida e descida da massa efetiva do corpo em cada passo foi considerado e a posição da carga dinâmica foi alterada de acordo com a posição do pedestre. Assim, a função de tempo, correspondente a excitação dinâmica induzida pela caminhada, possui uma variação espacial e temporal. O modelo tem a intenção de representar de modo mais realista os passos dados em uma caminhada. Contudo, é necessário estudar outros parâmetros neste tipo de modelagem numérica, tais como: à distância e a velocidade do passo. Esses parâmetros estão associados com a frequência do passo humano e são ilustrados pela Tabela 1.

Tabela 1. Características do caminhar humano [2].

\begin{tabular}{lccc}
\hline \multicolumn{1}{c}{ Atividade } & Velocidade $(\mathrm{m} / \mathrm{s})$ & Distância do Passo $(\mathrm{m})$ & Frequência do Passo \\
\hline Caminhada lenta & 1,1 & 0,6 & 1,7 \\
Caminhada normal & 1,5 & 0,75 & 2,0 \\
Caminhada rápida & 2,2 & 1,0 & 2,3 \\
\hline
\end{tabular}

O modelo matemático adotado no presente estudo foi proposto a partir de uma aproximação matemática respaldada por estudos experimentais que permitiram registrar a reação total de um piso, gerada ao longo do tempo, durante uma caminhada sobre plataformas rígidas [6,9], de acordo com a Fig. 3. 
Assim sendo, a carga dinâmica associada ao caminhar humano, considerando-se a incorporação do efeito do impacto do calcanhar, é obtida a partir das Eqs. (1) a (4) [6]. A função matemática proposta, Eqs. (1) a (4), utilizada para representar a carga dinâmica produzida por uma pessoa caminhando sobre um piso, não é simulada simplesmente por uma série de Fourier, pois a equação também incorpora em sua formulação, o pico transiente representativo do impacto do calcanhar sobre o piso.

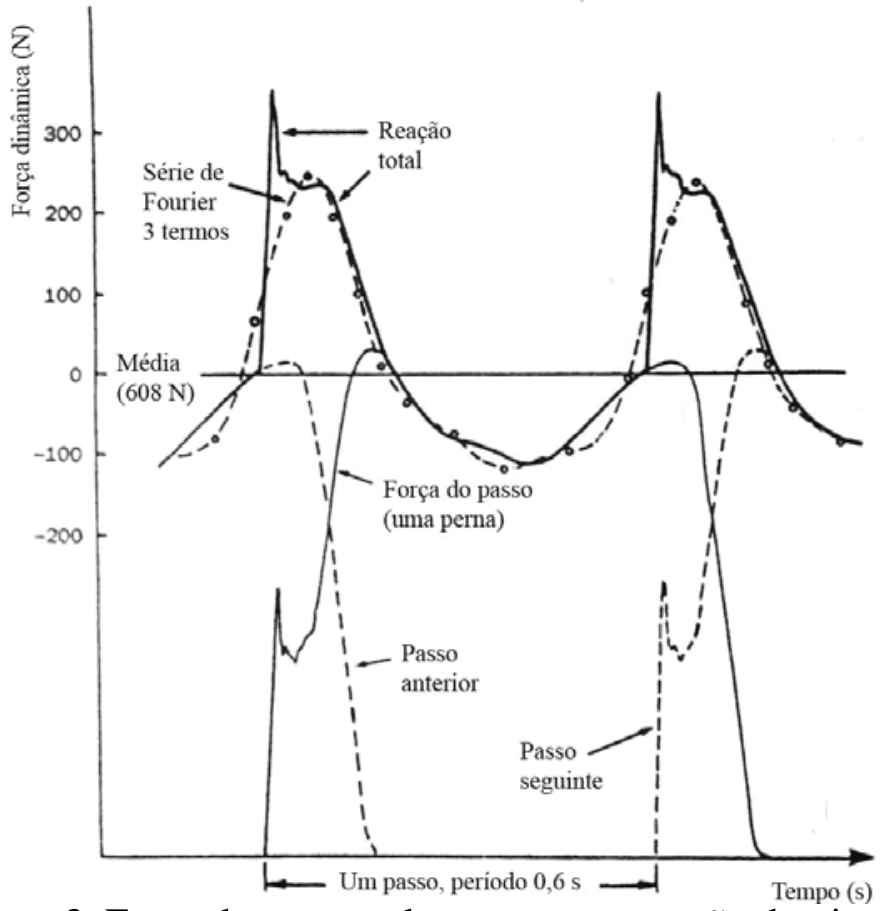

Figura 3. Força de contato de um passo e reação do piso [6].

$$
F(t)= \begin{cases}\left(\frac{f_{m i} F_{m}-P}{0,04 T_{p}}\right) t+P & \text { se } 0 \leq t<0,04 T_{p} \\ f_{m i} F_{m}\left[\frac{C_{1}\left(t-0,04 T_{p}\right)}{0,02 T_{p}}+1\right] & \text { se } 0,04 T_{p} \leq t<0,06 T_{p} \\ F_{m} & \text { se } 0,06 T_{p} \leq t<0,15 T_{p} \\ P+\sum_{i=1}^{n h} P \alpha \operatorname{sen}\left[2 \pi i f_{p}\left(t+0,1 T_{p}\right)+\phi_{i}\right] & \text { se } 0,15 T_{p} \leq t<0,90 T_{p} \\ 10\left(P-C_{2}\right) \cdot\left(\frac{t}{T_{p}}-1\right)+P & \text { se } 0,90 T_{p} \leq t<T_{p}\end{cases}
$$




$$
\begin{gathered}
C_{1}=\left(\frac{1}{f_{m i}}-1\right) \\
C_{2}= \begin{cases}P\left(1-\alpha_{2}\right) & \text { se } n h=3 \\
P\left(1-\alpha_{2}+\alpha_{4}\right) & \text { se } n h=4\end{cases}
\end{gathered}
$$

Onde:

$F_{m} \quad$ : valor máximo da série de Fourier, dado pela Eq. (2);

$f_{m i}$ : fator de majoração do impacto do calcanhar, $\left(f_{m i}=1,12\right)$, [6];

$T_{p} \quad$ : período do passo;

$C_{1}, C_{2}$ : coeficientes dados pelas Eqs. (3) e (4).

Na presente investigação, são empregadas as Eqs. (1) a (4) para obtenção da excitação dinâmica, proveniente dos seres humanos caminhando sobre os pisos; e se adota ainda, para o fator de amplificação do impacto do calcanhar humano, o valor de $1,12\left(f_{m i}=1,12\right)$, [6]. Todavia, faz-se necessário enfatizar que este valor de 1,12 varia de pessoa para pessoa fato que motiva que o referido coeficiente mereça um estudo mais aprofundado.

Assim sendo, de forma a demonstrar a modelagem numérica da ação de caminhar do pedestre sobre a estrutura, considera-se, como exemplo, uma das passarelas investigadas, com vão de $25 \mathrm{~m}$ e frequência fundamental igual a $5,7 \mathrm{~Hz}\left(f_{01}=5,7 \mathrm{~Hz}\right)$. Desta maneira, utiliza-se uma frequência do passo igual a $2 \mathrm{~Hz}\left(f_{p}=2 \mathrm{~Hz}\right)$, de acordo com a Tabela 1 , de forma a induzir a estrutura o mais próximo possível do fenômeno físico da ressonância ( 3 x 2Hz = 6Hz).

Nesta situação, a malha de elementos finitos do modelo precisa ser refinada suficientemente e, bem como, o tempo de contato da aplicação da carga dinâmica com a estrutura depende da distância e da frequência do passo, conforme apresentado na Tabela 1. Assim sendo, a distância do passo associada à frequência de passo de $2 \mathrm{~Hz}$ é igual a $0,75 \mathrm{~m}$. Na sequência da modelagem, a carga dinâmica associada ao caminhar do pedestre sobre a passarela, ao longo do tempo, é obtida mediante o emprego de quatro harmônicos utilizados para gerar a função de carregamento dinâmico, de acordo com a Tabela 2.

Tabela 2. Frequências do passo e coeficientes dinâmicos para os harmônicos da excitação [5].

\begin{tabular}{cccc}
\hline \multirow{2}{*}{ Harmônico i } & $\begin{array}{c}\text { Frequência do Passo } \\
\left(f_{p}\right)(\mathrm{Hz})\end{array}$ & $\begin{array}{c}\text { Coeficiente Dinâmico } \\
\left(\alpha_{i}\right)\end{array}$ & $\begin{array}{c}\text { Ângulo de Fase } \\
\left(\phi_{i}\right)\end{array}$ \\
\hline 1 & $1,6-2,2$ & 0,5 & 0 \\
2 & $3,2-4,4$ & 0,2 & $\pi / 2$ \\
3 & $4,8-6,6$ & 0,1 & $\pi$ \\
4 & $6,4-8,8$ & 0,05 & $3 \pi / 2$ \\
\hline
\end{tabular}

Deste modo, o período do passo é igual a $1 / f_{p}=1 / 2 \mathrm{~Hz}=0,5 \mathrm{~s}$, correspondente a uma distância de $0,75 \mathrm{~m}$. Portanto, adotando-se uma malha com elementos finitos com comprimento igual a $0,25 \mathrm{~m}$, seriam necessárias quatro cargas para representar um passo do pedestre sobre a passarela. Cada uma dessas cargas P1, P2, P3 e P4 seria aplicada durante 0,5/4 = 0,1667s, Figs. 4 e 5. 
Destaca-se que a ação dinâmica não é aplicada simultaneamente. A carga $P 1$ é aplicada durante $0,1667 \mathrm{~s}$, e no final deste período de tempo, $P 1$ assume um valor nulo e, logo em seguida, a carga $P 2$ é aplicada por 0,1667s. Este processo repete-se sucessivamente, ao longo do tempo, até o pedestre cruzar a estrutura. A ação dinâmica de caminhar é aplicada, de forma correta, ao longo de toda a passarela, Figs. 4 e 5.

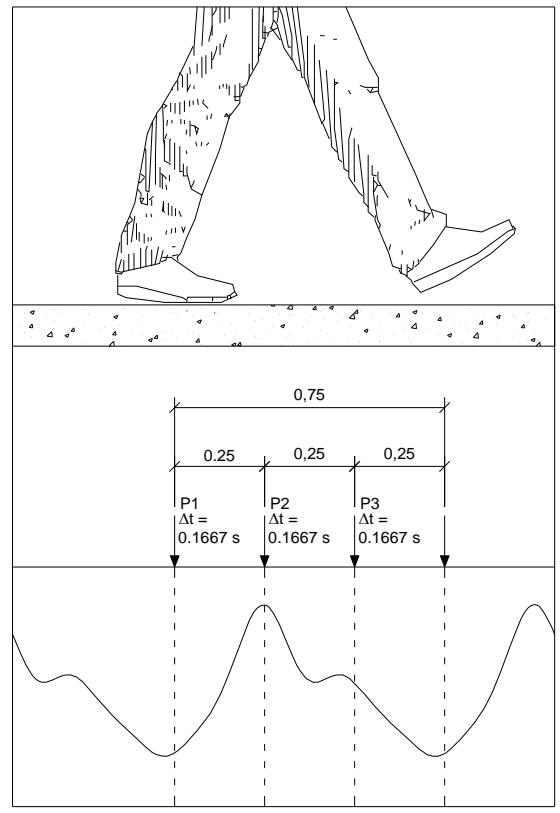

Figura 4. Representação do passo do pedestre sobre a passarela (dimensões em m).

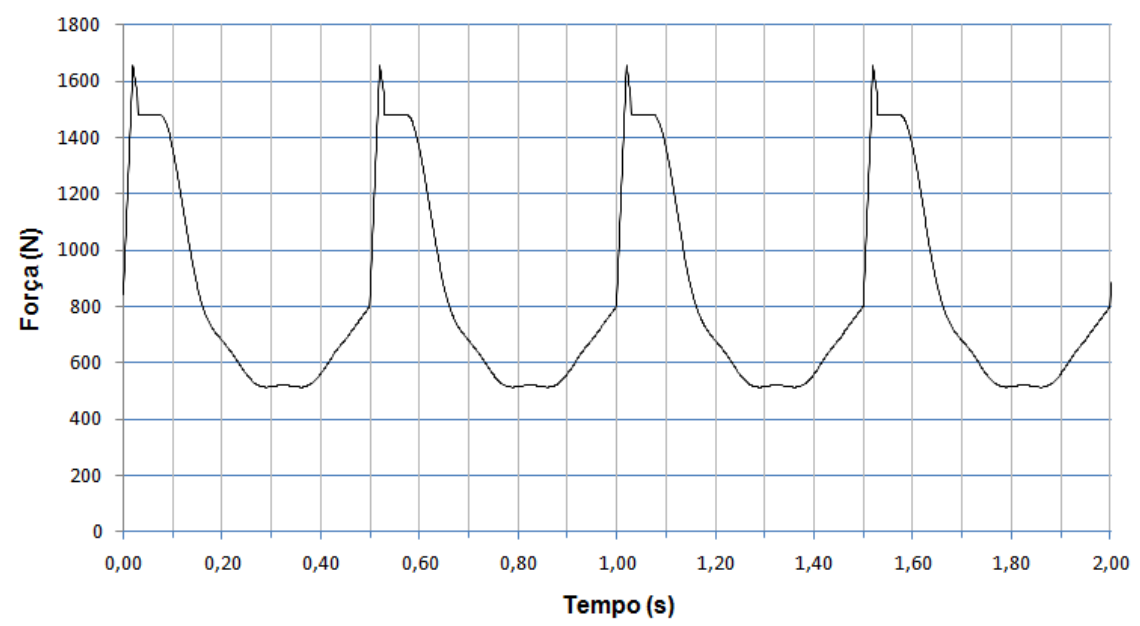

Figura 5. Função de carregamento dinâmico para uma caminhada com $\mathrm{f}_{\mathrm{p}}=1,9 \mathrm{~Hz}$.

\section{SISTEMA ESTRUTURAL INVESTIGADO}

Os modelos estruturais investigados correspondem a passarelas mistas com $10 \mathrm{~m}$ a 30m de extensão. O sistema estrutural utilizado para a travessia de pedestres é composto por perfis metálicos de seção "I" e um tabuleiro de concreto armado com espessura de $0,10 \mathrm{~m}$ ( $h=$ $100 \mathrm{~mm}$ ), apoiado sobre as vigas de aço, Fig. 6. A Tabela 3 apresenta as características geométricas dos perfis de aço empregados nas vigas do modelo da passarela com $25 \mathrm{~m}$ de vão. As propriedades dos demais perfis de aço podem ser obtidas no trabalho de Feitosa [1]. 
Adota-se o peso do indivíduo como sendo igual a $800 \mathrm{~N}(0,8 \mathrm{kN})$ e o amortecimento estrutural igual a $0,001(\xi=1 \%)$ [5]. O perfil metálico adotado possui tensão de escoamento $\left(f_{y}\right)$ e tensão de ruptura $\left(f_{u}\right)$ iguais a $250 \mathrm{MPa}$ e $400 \mathrm{MPa}$, respectivamente. O Módulo de Young foi adotado igual a 2,05 x $10^{5} \mathrm{MPa}$. A laje de concreto tem uma resistência à compressão de $30 \mathrm{MPa}$ com Módulo de Young igual a 3,0 x 104 MPa.

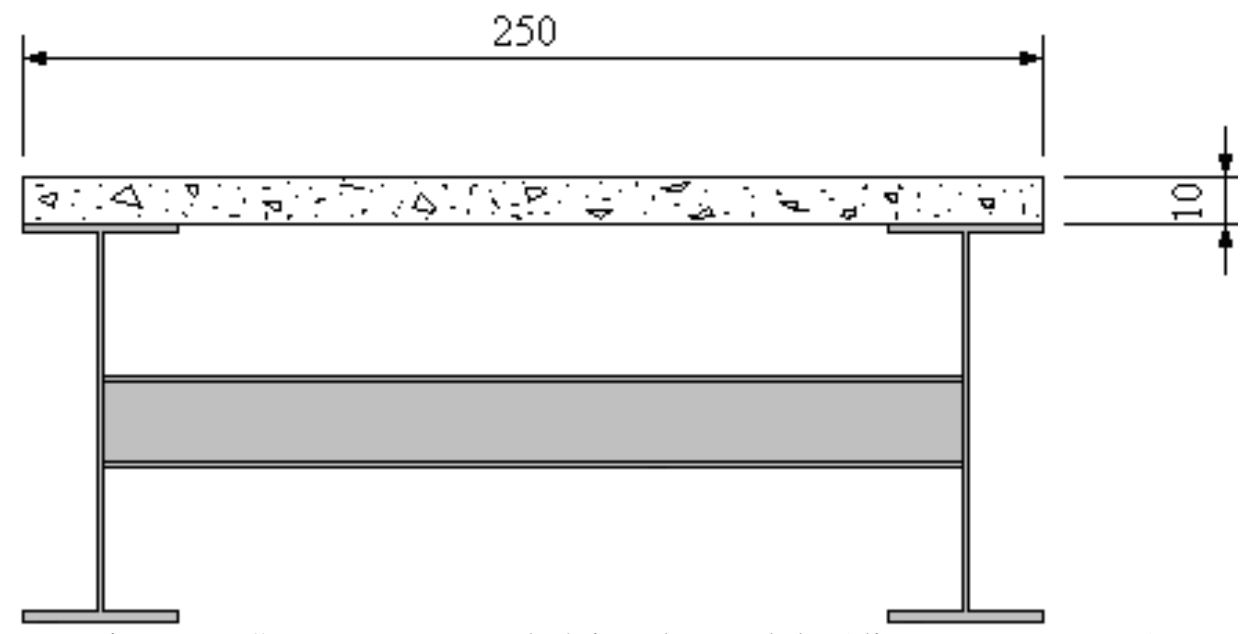

Figura 6. Seção transversal típica do modelo (dimensões em cm).

Tabela 3. Características geométricas dos perfis de aço das vigas do modelo estrutural.

Massa Altura do Largura da Espessura Espessura da Número Diâmetro Tipo do Perfil linear $(G)$ perfil $(\mathrm{h})$ mesa $\left(\mathrm{b}_{\mathrm{f}}\right)$ da mesa $\left(\mathrm{t}_{\mathrm{f}}\right)$ alma $\left(\mathrm{t}_{\mathrm{w}}\right)$ de furos do furo $\left(\mathrm{d}_{0}\right)$

\begin{tabular}{cccccccc}
\cline { 2 - 7 } & $\mathrm{kg} / \mathrm{m}$ & $\mathrm{mm}$ & $\mathrm{mm}$ & $\mathrm{mm}$ & $\mathrm{mm}$ & - & $\mathrm{mm}$ \\
\hline $\begin{array}{c}\text { Perfil de alma } \\
\text { cheia }\end{array}$ & 190,8 & 900 & 350 & 25 & 8 & - & - \\
$\begin{array}{c}\text { Perfil celular } \\
\text { VS 1305x181 }\end{array}$ & 181,3 & 1305 & 350 & 25 & 8 & 16 & 1044 \\
$\begin{array}{c}\text { Perfil celular } \\
\text { VS 1305x181 }\end{array}$ & 181,3 & 1305 & 350 & 25 & 8 & 31 & 743,85 \\
\hline
\end{tabular}

\section{MODELO EM ELEMENTOS FINITOS}

No modelo numérico-computacional desenvolvido para a análise dinâmica das passarelas são empregadas técnicas usuais de refinamento, via método dos elementos finitos, por meio do emprego do programa ANSYS [7]. No presente modelo, as vigas de aço são simuladas por elementos finitos de casca tridimensionais (SHELL63), onde são considerados os efeitos de flexão e de torção. A laje de concreto armado é simulada por meio de elementos finitos sólidos (SOLID45). Considera-se que o aço e o concreto possuem interação completa e que ambos trabalham no regime linear-elástico. A Fig. 7 ilustra o modelo em elementos finitos desenvolvido, com base no modelo estrutural com $25 \mathrm{~m}$ de vão para viga de alma cheia e celular, respectivamente. 


\section{ESTUDO DO COMPORTAMENTO DINÂMICO}

A resposta dinâmica das passarelas é determinada mediante a obtenção das frequências naturais, deslocamentos e acelerações máximas (acelerações de pico). Os resultados são obtidos através de uma análise numérica, com base no método dos elementos finitos, utilizando o programa ANSYS 12.0 [7]. Ressalta-se que as acelerações verticais máximas da passarela são calculadas e comparadas com os limites propostos por normas e recomendações de projeto [5]. Tal comparação é feita objetivando verificar a ocorrência de níveis de vibrações indesejáveis e, bem como, o desconforto humano.

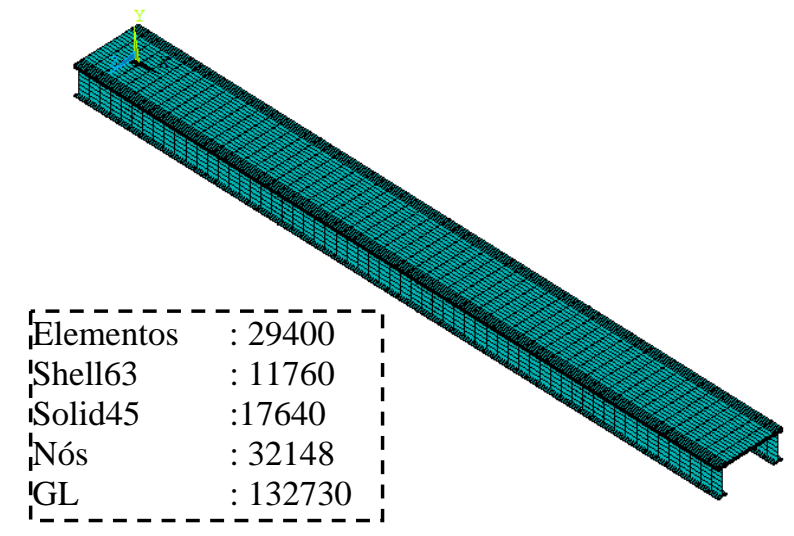

(a) Perfil de alma cheia

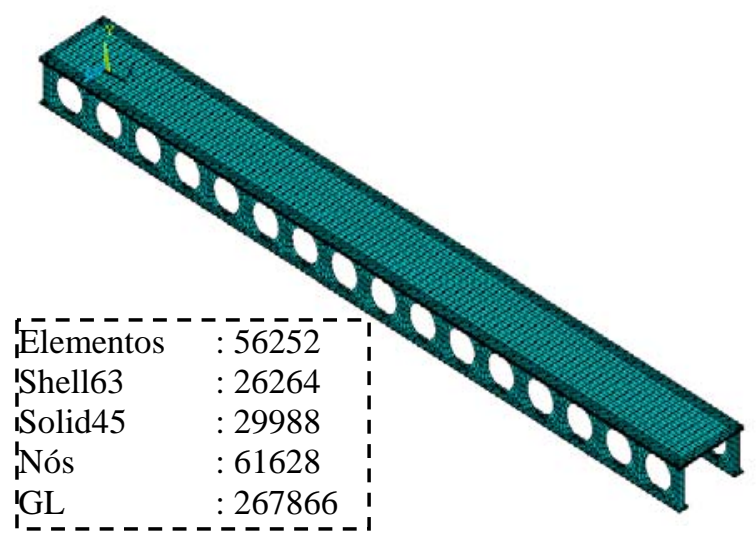

(b) Perfil celular

Figura 7. Modelos em elementos finitos desenvolvidos para a passarela com vão de $25 \mathrm{~m}$.

\subsection{Análise das frequências naturais e dos modos de vibração}

Com base nas simulações numéricas realizadas ao longo do estudo, são determinadas as frequências naturais das passarelas mistas (aço-concreto) com dimensões variando entre 10m e 30m, como apresentado na Tabela 4. Tal análise foi feita para os modelos de vigas com alma cheia (sem abertura na alma) e celulares (com aberturas circulares na alma das vigas), objetivando investigar, quantitativamente, qual a variação do valor da frequência fundamental das passarelas, no que diz respeito ao número de furos e, bem como, ao tamanho da abertura. Esta análise é de suma importância, pois os autores do trabalho desconhecem na literatura técnica disponível sobre o tema, qualquer avaliação desta natureza, e que pode influir diretamente no estudo de conforto humano das estruturas investigadas. 
Tabela 4. Frequência natural das passarelas (perfis com vão de 10 a 22,5 metros).

\begin{tabular}{|c|c|c|c|c|c|c|c|c|c|}
\hline \multirow{2}{*}{$\begin{array}{l}\text { Vão } \\
(\mathrm{m})\end{array}$} & \multirow{2}{*}{ Tipo de Perfil } & \multicolumn{6}{|c|}{ Frequências Naturais $f_{o i}(\mathrm{~Hz})$ [7] } & \multirow{2}{*}{$\begin{array}{l}\text { AISC* } \\
f_{01}(\mathrm{~Hz})\end{array}$} & \multirow{2}{*}{$\begin{array}{c}\Delta \\
(\%)\end{array}$} \\
\hline & & $f_{01}$ & $f_{02}$ & $f_{03}$ & $f_{04}$ & $f_{05}$ & $f_{06}$ & & \\
\hline \multirow{3}{*}{10,0} & $\begin{array}{c}\text { Perfil de Alma Cheia } \\
\text { VS 400x53 }\end{array}$ & 12,65 & 23,46 & 25,47 & 39,70 & 45,48 & 49,44 & 12,53 & $0,96 \%$ \\
\hline & $\begin{array}{c}\text { Perfil Celular } \\
\text { VS 580x51 (14 furos) }\end{array}$ & 12,86 & 22,29 & 24,81 & 36,71 & 42,93 & 44,23 & 12,76 & $0,78 \%$ \\
\hline & $\begin{array}{c}\text { Perfil Celular } \\
\text { VS 580x51 (28 furos) }\end{array}$ & 9,90 & 17,20 & 20,04 & 31,06 & 33,94 & 40,26 & 9,93 & $0,30 \%$ \\
\hline \multirow{3}{*}{12,5} & $\begin{array}{l}\text { Perfil de Alma Cheia } \\
\text { VS 500x73 }\end{array}$ & 10,84 & 20,38 & 23,04 & 39,00 & 44,13 & 44,37 & 10,77 & $0,65 \%$ \\
\hline & $\begin{array}{c}\text { Perfil Celular } \\
\text { VS 725x69 (14 furos) }\end{array}$ & 11,65 & 19,84 & 23,11 & 35,48 & 38,94 & 38,96 & 11,60 & $0,43 \%$ \\
\hline & $\begin{array}{c}\text { Perfil Celular } \\
\text { VS 725x69 (28 furos) }\end{array}$ & 9,31 & 15,57 & 18,91 & 29,63 & 30,84 & 38,27 & 9,35 & $0,43 \%$ \\
\hline \multirow{3}{*}{15,0} & $\begin{array}{l}\text { Perfil de Alma Cheia } \\
\text { VS 550x100 }\end{array}$ & 8,84 & 17,10 & 19,93 & 36,05 & 37,24 & 42,00 & 8,82 & $0,22 \%$ \\
\hline & $\begin{array}{c}\text { Perfil Celular } \\
\text { VS 798x95 (15 furos) }\end{array}$ & 9,76 & 16,61 & 20,28 & 32,20 & 32,76 & 32,78 & 9,75 & $0,10 \%$ \\
\hline & $\begin{array}{c}\text { Perfil Celular } \\
\text { VS 798x95 (31 furos) }\end{array}$ & 7,66 & 12,63 & 16,06 & 25,23 & 25,72 & 31,94 & 7,73 & $0,91 \%$ \\
\hline \multirow{3}{*}{17,5} & $\begin{array}{l}\text { Perfil de Alma Cheia } \\
\text { VS 600x140 }\end{array}$ & 7,51 & 14,90 & 18,02 & 32,96 & 35,11 & 42,51 & 7,51 & $0,00 \%$ \\
\hline & $\begin{array}{c}\text { Perfil Celular } \\
\text { VS 870x133 (16 furos) }\end{array}$ & 8,55 & 13,33 & 18,41 & 28,02 & 29,50 & 32,59 & 8,48 & $0,82 \%$ \\
\hline & $\begin{array}{c}\text { Perfil Celular } \\
\text { VS 870x133 (31furos) }\end{array}$ & 7,94 & 13,04 & 17,14 & 26,16 & 27,98 & 32,72 & 7,99 & $0,63 \%$ \\
\hline \multirow{3}{*}{20,0} & $\begin{array}{l}\text { Perfil de Alma Cheia } \\
\text { VS 700x154 }\end{array}$ & 6,77 & 13,57 & 16,44 & 30,41 & 33,14 & 41,19 & 6,77 & $0,00 \%$ \\
\hline & $\begin{array}{c}\text { Perfil Celular } \\
\text { VS 1015x146 (16 fu- }\end{array}$ & 7,74 & 12,07 & 16,84 & 25,18 & 25,48 & 25,55 & 7,69 & $0,65 \%$ \\
\hline & $\begin{array}{c}\text { Perfil Celular } \\
\text { VS 1015x146 (31 fu- }\end{array}$ & 6,75 & 10,72 & 14,44 & 21,39 & 23,48 & 25,33 & 6,81 & $0,88 \%$ \\
\hline \multirow{3}{*}{22,5} & $\begin{array}{l}\text { Perfil de Alma Cheia } \\
\text { VS } 800 \times 173\end{array}$ & 6,18 & 12,52 & 15,19 & 28,21 & 31,17 & 37,68 & 6,18 & $0,00 \%$ \\
\hline & $\begin{array}{c}\text { Perfil Celular } \\
\text { VS 1160x164 (16 fu- }\end{array}$ & 7,03 & 10,98 & 15,46 & 20,99 & 21,06 & 22,61 & 7,00 & $0,42 \%$ \\
\hline & $\begin{array}{c}\text { Perfil Celular } \\
\text { VS 1160x164 (31 fu- }\end{array}$ & 6,13 & 9,73 & 13,27 & 19,44 & 21,03 & 21,07 & 6,19 & 0,97\% \\
\hline
\end{tabular}


Tabela 5. Frequência natural das passarelas (perfis com vão de 25 a 30 metros).

\begin{tabular}{|c|c|c|c|c|c|c|c|c|c|}
\hline \multirow{2}{*}{$\begin{array}{l}\text { Vão } \\
(\mathrm{m})\end{array}$} & \multirow{2}{*}{ Tipo de Perfil } & \multicolumn{6}{|c|}{ Frequências Naturais $f_{0 i}(\mathrm{~Hz})$ (ANSYS, } & \multirow{2}{*}{$\begin{array}{l}\text { AISC* } \\
f_{01}(\mathrm{~Hz})\end{array}$} & \multirow{2}{*}{$\begin{array}{c}\Delta \\
(\%)\end{array}$} \\
\hline & & $f_{01}$ & $f_{02}$ & $f_{03}$ & $f_{04}$ & $f_{05}$ & $f_{06}$ & & \\
\hline \multirow{3}{*}{25,0} & $\begin{array}{l}\text { Perfil de Alma Cheia } \\
\text { VS 900x191 }\end{array}$ & 5,70 & 11,34 & 14,08 & 25,79 & 25,69 & 29,19 & 5,68 & $0,35 \%$ \\
\hline & $\begin{array}{c}\text { Perfil Celular } \\
\text { VS 1305x181 (16 furos) }\end{array}$ & 6,40 & 10,06 & 14,39 & 18,89 & 19,01 & 18,22 & 6,38 & $0,31 \%$ \\
\hline & $\begin{array}{c}\text { Perfil Celular } \\
\text { VS 1305x181 (31 furos) }\end{array}$ & 5,56 & 8,66 & 12,04 & 16,81 & 16,85 & 17,26 & 5,61 & $0,90 \%$ \\
\hline \multirow{3}{*}{27,5} & $\begin{array}{l}\text { Perfil de Alma Cheia } \\
\text { VS 1000x201 }\end{array}$ & 5,25 & 10,67 & 13,16 & 24,34 & 24,39 & 24,45 & 5,25 & $0,00 \%$ \\
\hline & $\begin{array}{c}\text { Perfil Celular } \\
\text { VS 1450x191 (15 furos) }\end{array}$ & 6,12 & 9,53 & 13,47 & 13,57 & 13,76 & 14,85 & 6,11 & $0,16 \%$ \\
\hline & $\begin{array}{c}\text { Perfil Celular } \\
\text { VS 1450x191 (30 furos) }\end{array}$ & 5,42 & 8,45 & 11,90 & 13,54 & 13,60 & 15,02 & 5,47 & $0,92 \%$ \\
\hline \multirow{3}{*}{30,0} & $\begin{array}{l}\text { Perfil de Alma Cheia } \\
\text { VS 1100x235 }\end{array}$ & 4,92 & 10,23 & 12,51 & 23,53 & 27,09 & 27,20 & 4,91 & $0,20 \%$ \\
\hline & $\begin{array}{c}\text { Perfil Celular } \\
\text { VS 1595x224 (15 furos) }\end{array}$ & 5,86 & 9,20 & 13,34 & 13,79 & 13,93 & 14,86 & 5,85 & $0,17 \%$ \\
\hline & $\begin{array}{c}\text { Perfil Celular } \\
\text { VS 1595x224 (30 furos) }\end{array}$ & 5,20 & 8,09 & 11,53 & 13,92 & 14,00 & 14,99 & 5,25 & $0,96 \%$ \\
\hline
\end{tabular}

Os resultados apresentados na Tabela 4 indicam diferenças numéricas que podem vir a ser importantes, no que diz respeito ao valor da frequência fundamental das passarelas, em todos os vãos investigados, quando são comparados os casos entre perfis de alma cheia e celulares (número mínimo e máximo de furos). As diferenças entre estes valores chegam a 20\% (diferença máxima), Tabela 4. Estas diferenças também são ilustradas pelas Figs. 8 a 13, onde são apresentadas as curvas formadas pela variação das frequências fundamentais $\left(f_{01}\right)$ das passarelas de perfil celular, de acordo com a variação dos diâmetros dos furos e com a flexibilidade permitida [8].

As Figs. 14 a 16 ilustram os modos de vibração correspondentes às três primeiras frequências naturais do modelo estrutural (alma cheia e celular) com vão de $25 \mathrm{~m}$. Observou-se que nos modelos estruturais investigados os efeitos de flexão foram predominantes no modo fundamental de vibração. No entanto, é importante salientar que os efeitos de torção estão presentes a partir do segundo modo de vibração da estrutura, Figs. 14 a 16.

Destaca-se ainda, que os resultados numéricos [7], associados à frequência fundamental das passarelas estão de acordo com os valores calculados por meio do guia de projeto do AISC [5], demonstrando que os modelos numéricos estão coerentes com a solução analítica. Deve-se destacar que durante as análises, foram avaliados os comportamentos dos perfis de acordo com a variação do diâmetro do furo, Fig 8 a 13. 


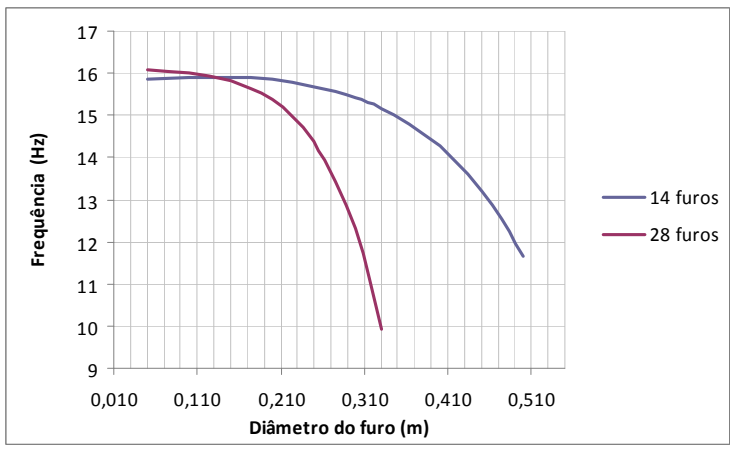

Figura 8. Variação da frequência $\left(f_{01}\right)$ da passarela celular com vão de10m.

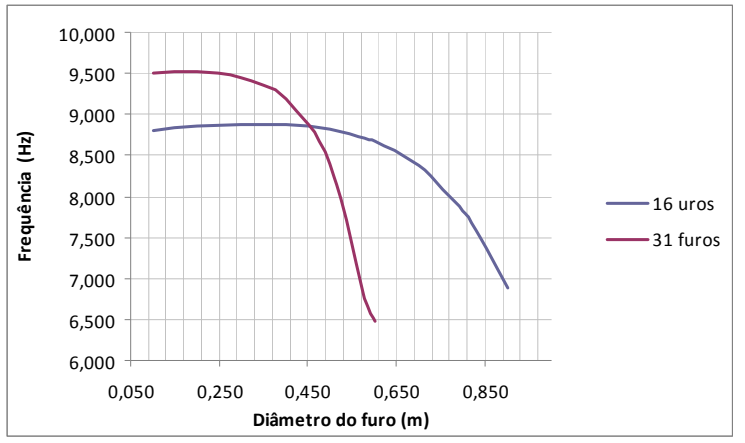

Figura 10: Variação da frequência $\left(f_{01}\right)$ da passarela celular com vão de20m.

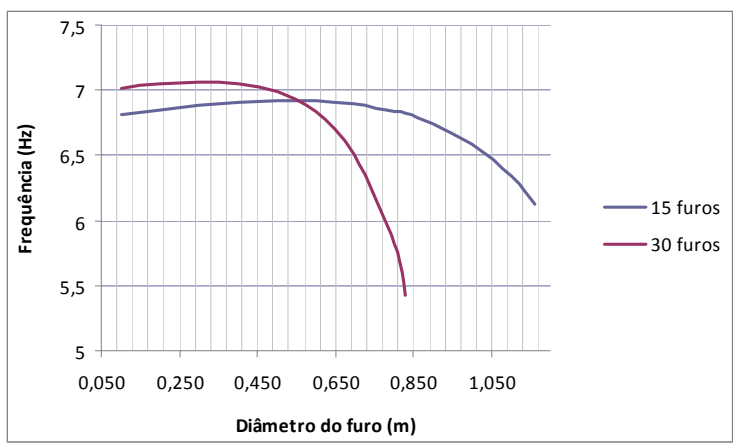

Figura 12: Variação da frequência $\left(f_{01}\right)$ da passarela celular com vão de 27,5m.

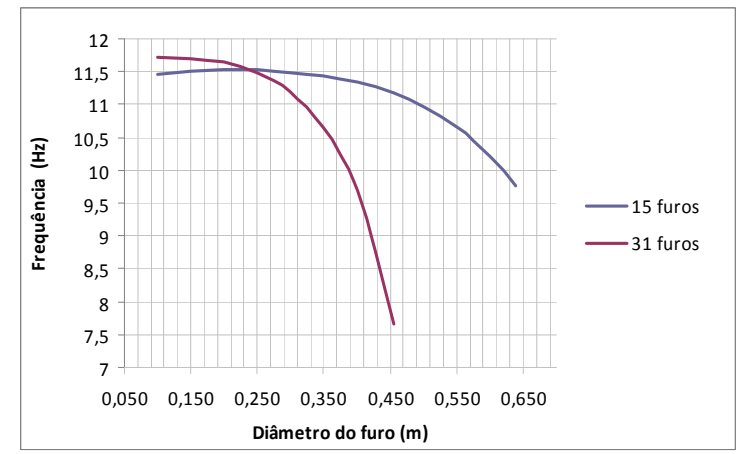

Figura 9. Variação da frequência $\left(f_{01}\right)$ da passarela celular com vão de15m.

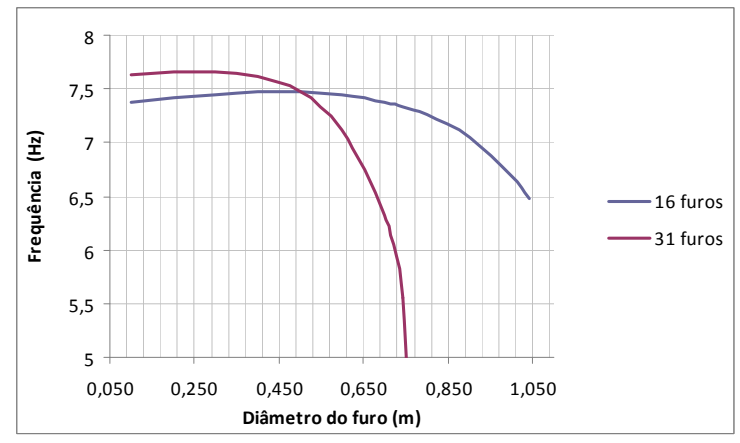

Figura 11: Variação da frequência $\left(f_{01}\right)$ da passarela celular com vão de25m.

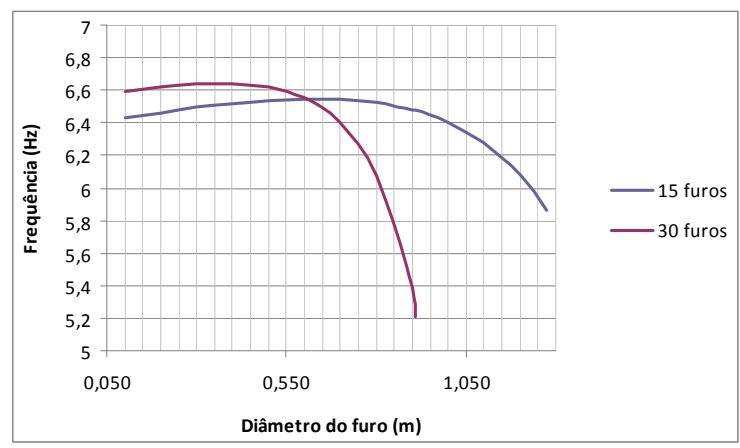

Figura 13: Variação da frequência $\left(f_{01}\right)$ da passarela celular com vão de $30 \mathrm{~m}$.

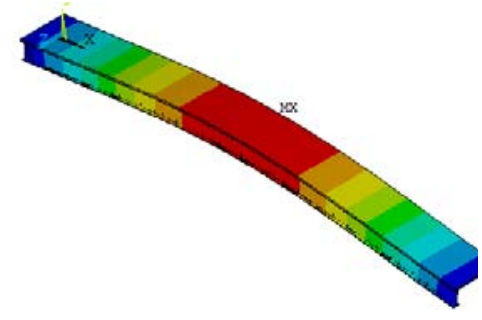

$1^{\circ}$ Modo de vibração $\left(\mathrm{f}_{01}=5,70 \mathrm{~Hz}\right)$

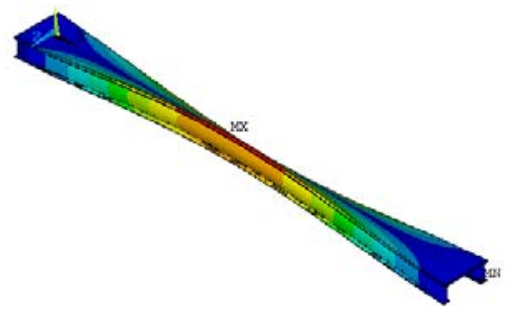

$2^{\circ}$ Modo de vibração $\left(\mathrm{f}_{02}=11,34 \mathrm{~Hz}\right)$

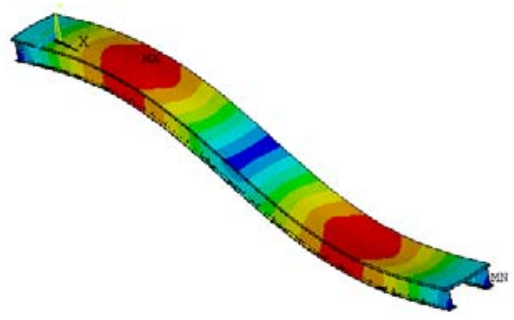

$3^{\circ}$ Modo de vibração $\left(\mathrm{f}_{03}=14,08 \mathrm{~Hz}\right)$

Figura 14. Modos de vibração da passarela (L=25m). Perfil sólido: VS 1100x235. 


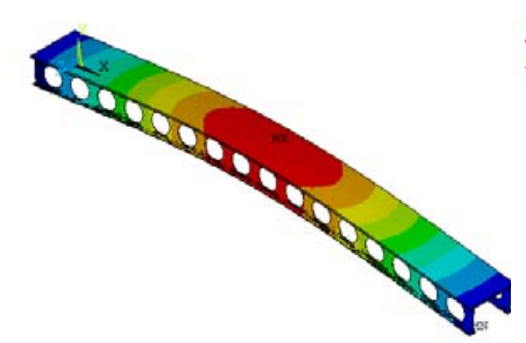

$1^{\circ}$ Modo de vibração

$$
\left(\mathrm{f}_{01}=6,40 \mathrm{~Hz}\right)
$$

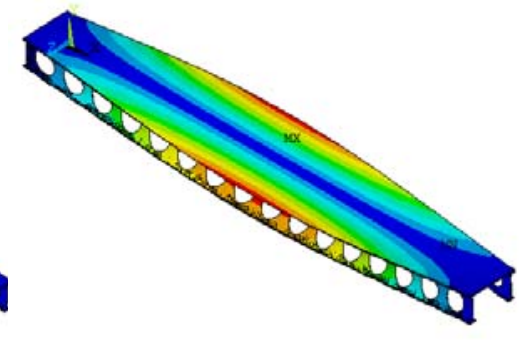

$2^{\circ}$ Modo de vibração

$\left(\mathrm{f}_{02}=10,06 \mathrm{~Hz}\right)$

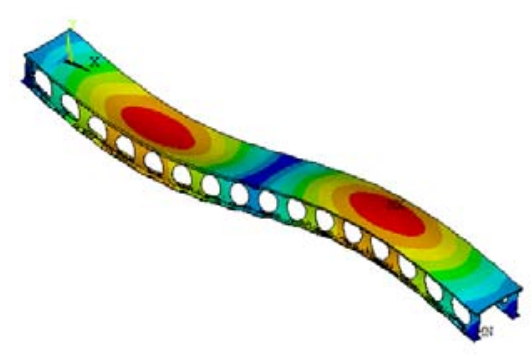

$3^{\circ}$ Modo de vibração $\left(\mathrm{f}_{03}=14,39 \mathrm{~Hz}\right)$

Figura 15. Modos de vibração da passarela (L=25m). Perfil celular: VS 1595x224 (15 furos).

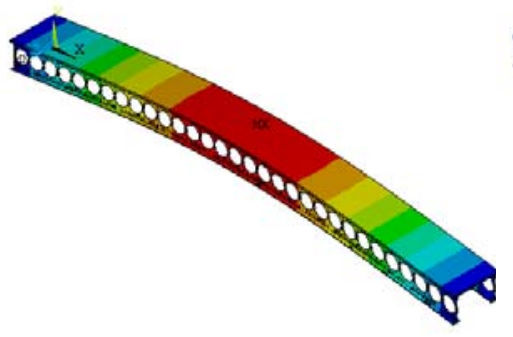

$1^{\circ}$ Modo de vibração

$\left(\mathrm{f}_{01}=5,56 \mathrm{~Hz}\right)$

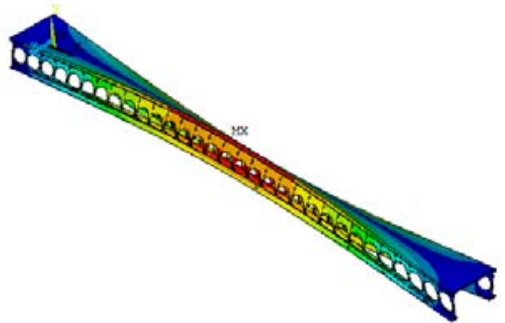

$2^{\circ}$ Modo de vibração

$\left(\mathrm{f}_{02}=8,66 \mathrm{~Hz}\right)$

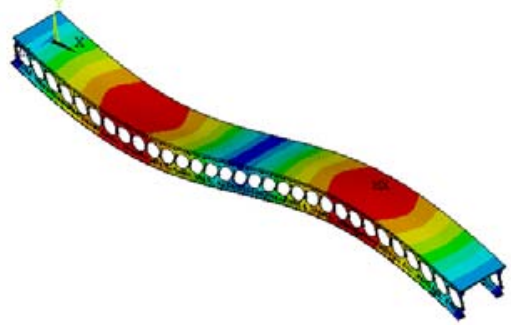

$3^{\circ}$ Modo de vibração

$\left(\mathrm{f}_{03}=12,04 \mathrm{~Hz}\right)$

Figura 16. Modos de vibração da passarela (L=25m). Perfil celular: VS 1595x224 (30 furos).

\subsection{Análise das acelerações de pico}

Nesta seção, pretende-se verificar a adequabilidade da passarela quando submetida ao caminhar humano, no que tange ao desconforto relacionado a vibrações. Deste modo, o modelo de carregamento descrito anteriormente é aplicado como excitação dinâmica sobre a passarela de forma a determinar as acelerações de pico, de acordo com as Eqs. (1) a (4).

A Fig. 17 ilustra o caso de carregamento dinâmico investigado neste trabalho, correspondente a travessia de um pedestre cruzando a passarela com comprimento de vão de $25 \mathrm{~m}$ (modelo escolhido para análise), com base nos parâmetros associados ao caminhar normal, Tabela 1. Para tal foram considerados os perfis de alma cheia (sem aberturas na alma, Fig. 7) e celulares (com aberturas na alma: 16 furos e 31 furos, Fig. 7).

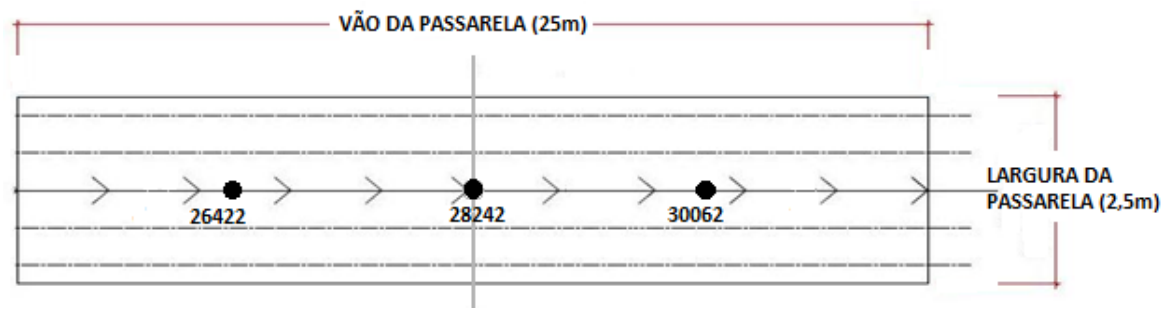

Figura 17. Planta baixa representativa do caso de carregamento investigado. 
Na sequência do estudo, as Figs. 18 e 19 ilustram a resposta dinâmica da passarela investigada (vão de $25 \mathrm{~m}$ ), considerando-se, inicialmente, os perfis de alma cheia, em termos da variação dos deslocamentos translacionais verticais e acelerações, ao longo do tempo. O ponto da estrutura analisado corresponde à seção do meio do vão do modelo (Nó: 28242, Fig. 17).

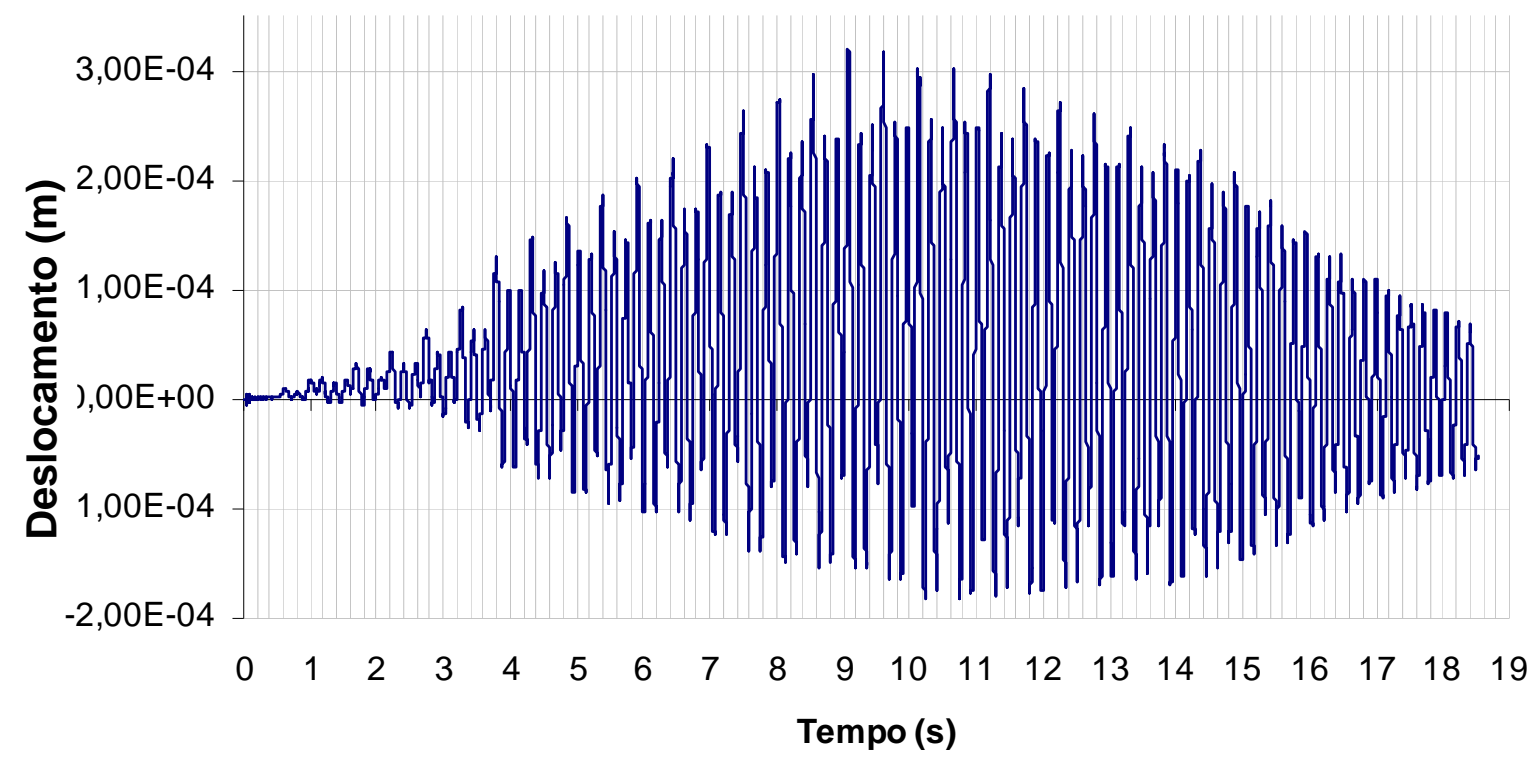

Figura 18. Deslocamento translacional vertical no meio do vão (Nó 28242).

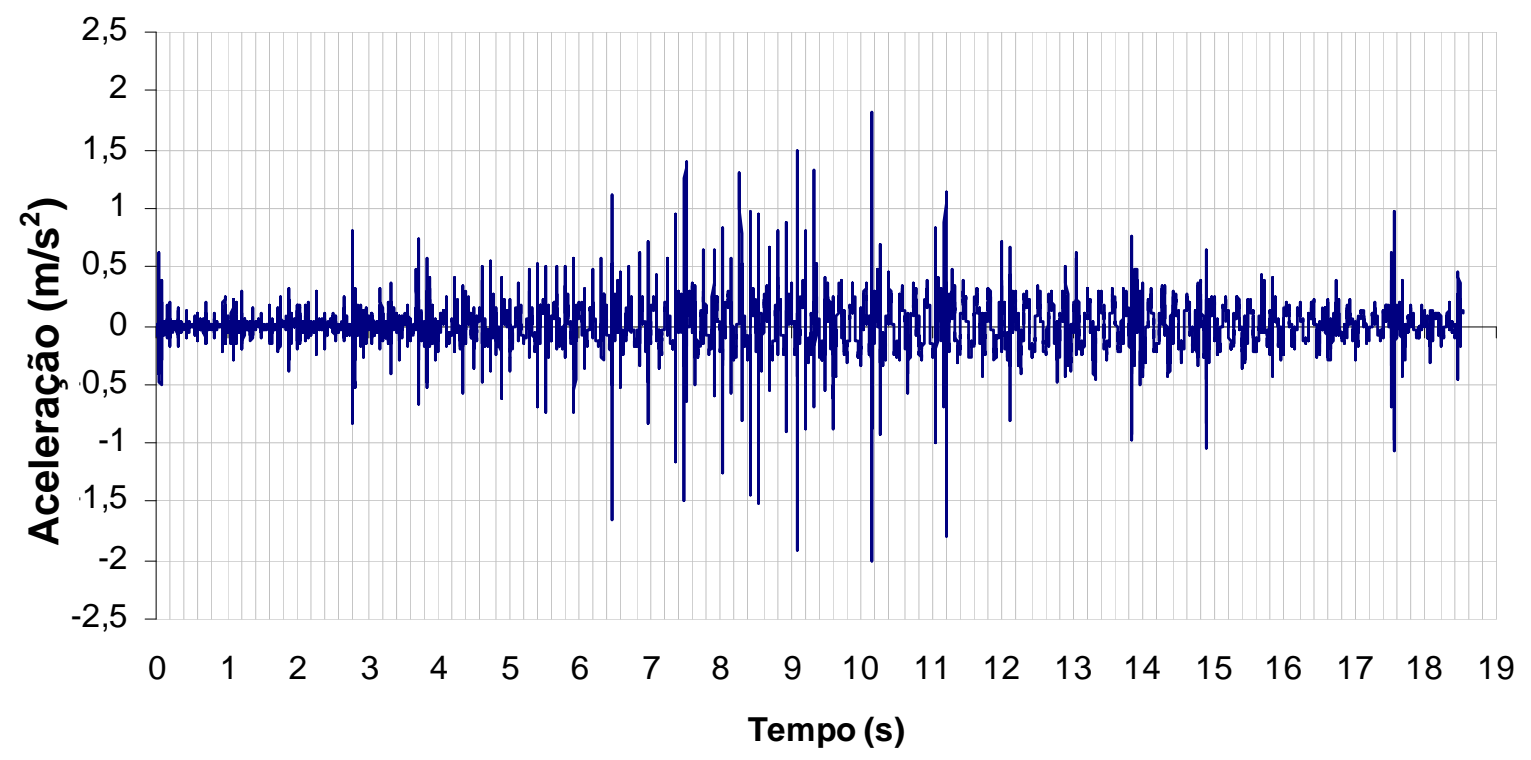

Figura 19. Aceleração no meio do vão (Nó 28242).

Em seguida, a Tabela 5 ilustra os valores numéricos das acelerações de pico encontradas, no que tange o modelo estrutural em estudo. A carga dinâmica relacionada a uma pessoa atravessando a passarela, através da linha de centro longitudinal da laje de concreto, de acordo com as Eqs. (1) a (4), foi aplicada durante um tempo de 18,56s. As acelerações de pico foram comparadas com os limites recomendados por normas e recomendações de projeto [5]. 
Tabela 5. Acelerações máximas do modelo estrutural.

\begin{tabular}{lcc}
\hline \multicolumn{1}{c}{$\begin{array}{c}\text { Comprimento do Vão } \\
(L=25 \mathrm{~m})\end{array}$} & $\begin{array}{c}\text { Impacto do } \\
\text { Calcanhar }\left(f_{m i}\right)\end{array}$ & $\begin{array}{c}\text { Acelerações de Pico* } \\
\left(a_{p} \text { em m/s } \mathrm{s}^{2}\right.\end{array}$ \\
\hline Perfil de alma cheia (VS 900x191) & 1,80 \\
Perfil celular (VS 1305x181) com 16 furos & 1.12 & 1,70 \\
Perfil celular (VS 1305x181) com 31 furos & 1,68 \\
$* a_{\text {lim }}=0,15 \mathrm{~m} / \mathrm{s}^{2}:$ passarelas internas [5]. & \\
$* a_{\text {lim }}=0,50 \mathrm{~m} / \mathrm{s}^{2}:$ passarelas externas [5]. & \\
\hline
\end{tabular}

Os resultados alcançados até o presente momento indicam que a proximidade da frequência fundamental dos três modelos investigados, perfil de alma cheia (VS 900x191) ( $f_{01}=$ $5,7 \mathrm{~Hz}$ ), perfil celular (VS $1305 \times 181)$ com 16 furos $\left(f_{01}=6,4 \mathrm{~Hz}\right)$ e perfil celular (VS $1305 \times 181)$ com 31 furos $\left(f_{01}=5,56 \mathrm{~Hz}\right)$, com a frequência do terceiro harmônico do passo do pedestre $\left(f_{p}=2 \mathrm{~Hz}: f_{p}=3 \times 2=6 \mathrm{~Hz}\right.$, Tabela 1$)$, demonstram que os valores de aceleração de pico ultrapassam os limites estabelecidos em normas de projeto [5], violando os critérios de conforto humano, no que diz respeito à seção central do modelo, Fig. 19 e Tabela 5. Verificase, também, que considerando-se as diferenças da ordem de $2 \%$ a $10 \%$ entre o valor da frequência fundamental dos três modelos analisados ( $\mathrm{L}=25 \mathrm{~m})$, a diferença final, no que diz respeito ao valor da aceleração de pico, apresenta-se muito pequena.

\section{CONSIDERAÇÕES FINAIS}

Esta investigação foi desenvolvida com o objetivo de avaliar o comportamento dinâmico de passarelas de pedestres mistas (aço-concreto), nas quais as vigas de aço possuem aberturas na alma de diferentes geometrias e formas. Pretendeu-se, portanto, verificar a real influência das aberturas nas almas dessas vigas metálicas sobre a resposta dinâmica de passarelas de pedestres.

Os modelos estruturais investigados correspondem a passarelas mistas (aço-concreto) com $10 \mathrm{~m}$ a $30 \mathrm{~m}$ de extensão. O sistema estrutural utilizado para a travessia de pedestres foi composto por perfis metálicos de seção "I" e um tabuleiro de concreto armado. A análise fundamenta-se na modelagem numérica dessas passarelas. Para tal, foram empregadas técnicas usuais de discretização, via utilização do programa ANSYS [7].

Uma avaliação crítica acerca da resposta dinâmica das passarelas indicou diferenças que podem vir a ser relevantes para o projeto estrutural, no que diz respeito ao valor da frequência fundamental dos modelos investigados, quando são comparados os casos entre perfis sólidos e celulares (número mínimo e máximo de furos). A diferença entre estes valores de frequência, no que tange às passarelas analisadas, pode chegar até 20\% (diferença máxima).

No que diz respeito à questão associada ao conforto humano, o valor máximo encontrado para a aceleração de pico foi da ordem de $1,8 \mathrm{~m} / \mathrm{s}^{2}$, enquanto que o valor máximo admi- 
tido é de $0,15 \mathrm{~m} / \mathrm{s}^{2}$ (passarelas internas) e $0,5 \mathrm{~m} / \mathrm{s}^{2}$ (passarelas ao ar livre). Os resultados indicaram que a passarela analisada (vão de $25 \mathrm{~m}$ ) viola os critérios do conforto humano, devido a proximidade da frequência fundamental dos três modelos investigados (perfis de alma cheia e celulares) com a frequência do terceiro harmônico da carga dinâmica [5].

\section{Agradecimentos}

Os autores agradecem ao suporte financeiro fornecido pelas Agências de Fomento à Pesquisa do país, CAPES, CNPq e FAPERJ, que possibilitaram a realização deste trabalho de pesquisa.

\section{REFERÊNCIAS BIBLIOGRÁFICAS}

[1] Feitosa, F. F. Análise dinâmica de passarelas de pedestres mistas (aço-concreto) compostas por vigas de aço com abertura na alma, Dissertação de Mestrado (Em andamento), Programa de Pós-Graduação em Engenharia Civil, PGECIV, Universidade Estadual do Rio de Janeiro, UERJ, Rio de Janeiro/RJ, Brasil, 2011

[2] Bachmann, H. e Ammann, W. Vibrations in structures induced by man and machines, Structural Engineering Document 3e, International Association for Bridges and Structural Engineering, 1987.

[3] Figueiredo, F.P. Estudo do comportamento dinâmico de passarelas devido ao caminhar de pedestres, Dissertação de Mestrado, Programa de Pós-Graduação em Engenharia Civil, PGECIV, Universidade do Estado do Rio de Janeiro, UERJ, Rio de Janeiro, Brasil, 2005.

[4] Lima, N.L. de A. Efeito da modelagem do carregamento, do impacto do calcanhar humano e do amortecimento estrutural na resposta dinâmica de passarelas mistas, Dissertação de Mestrado, Programa de Pós-Graduação em Engenharia Civil, PGECIV, Universidade do Estado do Rio de Janeiro, UERJ, Rio de Janeiro, Brasil, 2007.

[5] Murray, T.M., Allen, D.E., Ungar, E.E. Floor vibrations due to human activity, Steel Design Guide Series, American Institute of Steel Construction, AISC, Chicago, USA, 2003.

[6] Varela, W.D. Modelo teórico-experimental para análises de vibrações induzidas por pessoas caminhando sobre lajes de edifícios, Tese de Doutorado, Universidade Federal do Rio de Janeiro, Departamento de Engenharia Civil, COPPE/UFRJ, Rio de Janeiro, Brasil, 2004.

[7] ANSYS. Swanson Analysis Systems, Inc., P.O. Box 65, Johnson Road, Houston, PA, 15342-0065, Version 12.0, Basic analysis procedures, Second edition, 2009.

[8] Harper, C. S. Design in Steel 4: Castellated \& cellular beams, British Steel Publications, 1994.

[9] Ohlsson, S.V. Floor Vibration and Human Disconfort, PhD Thesis, Department of Structural Engineering, Chalmers University of Technology, Gotemborg, Sweden, 1982. 